\title{
BMJ Open How are decisions made in cancer care? A qualitative study using participant observation of current practice
}

\author{
Pola Hahlweg, ${ }^{1}$ Martin Härter, ${ }^{1}$ Yvonne Nestoriuc, ${ }^{2,3}$ Isabelle Scholl ${ }^{1}$
}

To cite: Hahlweg P, Härter M, Nestoriuc $Y$, et al. How are decisions made in cancer care? A qualitative study using participant observation of current practice. BMJ Open 2017;7:e016360. doi:10.1136/ bmjopen-2017-016360

- Prepublication history and additional material for this paper are available online. To view please visit the journal (http:// dx.doi.org/10.1136/bmjopen2017-016360).

Received 7 March 2017 Revised 27 July 2017 Accepted 24 August 2017

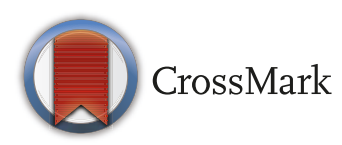

${ }^{1}$ Department of Medical Psychology, University Medical Center Hamburg-Eppendorf, Hamburg, Germany

2Department of Psychosomatic Medicine and Psychotherapy, University Medical Center Hamburg-Eppendorf, Hamburg, Germany

${ }^{3}$ Schön Klinik Hamburg Eilbek, Hamburg, Germany

Correspondence to Dr Pola Hahlweg; p.hahlweg@uke.de

\section{ABSTRACT}

Objectives Shared decision-making has continuously gained importance over the last years. However, few studies have investigated the current state of shared decision-making implementation in routine cancer care. This study aimed to investigate how treatment decisions are made in routine cancer care and to explore barriers and facilitators to shared decision-making using an observational approach (three independent observers). Furthermore, the study aimed to extend the understanding of current decision-making processes beyond the dyadic physician-patient interaction.

Design Cross-sectional qualitative study using participant observation with semistructured field notes, which were analysed using qualitative content analysis as described by Hsieh and Shannon.

Setting and participants Field notes from participant observations were collected at $n=54$ outpatient consultations and during two 1-week-long observations at two inpatient wards in different clinics of one comprehensive cancer centre in Germany.

Results Most of the time, either one physician alone or a group of physicians made the treatment decisions. Patients were seldom actively involved. Patients who were 'active' (ie, asked questions, demanded participation, opposed treatment recommendations) facilitated shared decisionmaking. Time pressure, frequent alternation of responsible physicians and poor coordination of care were the main observed barriers for shared decision-making. We found high variation in decision-making behaviour between different physicians as well as the same physician with different patients.

Conclusion Most of the time physicians made the treatment decisions. Shared decision-making was very rarely implemented in current routine cancer care. The entire decision-making process was not observed to follow the principles of shared decision-making. However, some aspects of shared decision-making were occasionally incorporated. Individual as well as organisational factors were found to influence the degree of shared decisionmaking. If future routine cancer care wishes to follow the principles of shared decision-making, strategies are needed to foster shared decision-making in routine cancer care.

\section{INTRODUCTION}

Patient-centred healthcare has gained importance over the last few years. A central

\section{Strengths and limitations of this study}

This study's main strength is the extensive qualitative exploration of decision-making processes in cancer care.

- The observation of patient-physician consultations as well as processes related to medical decisionmaking beyond the dyad of patient and physician further strengthens this study.

- Studies like this one are essential to inform implementation efforts to foster shared decisionmaking in cancer care.

- Further research is needed to explore whether our findings are generalisable to other institutions and countries.

- The use of a solely qualitative approach is a limitation.

dimension of patient-centred care is shared decision-making (SDM). ${ }^{1} \mathrm{SDM}$ is a process in which the physician and the patient both play an active role in making decisions. Each of them shares important information (ie, the physician shares medical knowledge and the patient shares his or her values, preferences and goals for care) and they subsequently come to a decision that both parties can agree on. ${ }^{23}$ In cancer care, SDM is especially relevant because in many cases several treatment options with different risks and benefits exist (ie, high level of preference-sensitivity), and treatments often have a considerable impact on patients' quality of life. ${ }^{24}$

Different stakeholders support SDM, and several studies have shown that the majority of patients wants to participate in treatment decision-making. ${ }^{5-7}$ There are current policy-related activities in many countries to foster SDM. ${ }^{3}$ In Germany, the National Cancer Plan, the patients' law from 2013 and clinical practice guidelines are advocating patient-centred care and SDM. ${ }^{8}{ }^{9}$ Additionally, SDM has been shown to be associated with patients being better informed and knowing more about potential risks and benefits of different options. ${ }^{10}$ As a result of those improvements, 
patients were more satisfied with the decision-making and the treatment processes. ${ }^{10}$

Nevertheless, SDM was found to be poorly implemented in routine care. ${ }^{11-14}$ Previous research focused on barriers and facilitators to understand why SDM is not easily transferred into routine care. In a systematic review of 38 studies, physicians reported time constraints as well as perceived lack of applicability for specific patients and for the clinical situation as main barriers. Healthcare provider (HCP) motivation was referred to as one main facilitator for SDM. ${ }^{15}$ Besides being well informed, patients also need to feel empowered to engage in SDM. ${ }^{16} \mathrm{~Pa}-$ tients still often report feeling caught in the traditional hierarchical structure and power imbalance between physicians and patients. ${ }^{17}$ Some studies used theoretical models, for example, the normalisation process theory, to explain why implementation of SDM is lagging. ${ }^{18}$ Elwyn and colleagues ${ }^{19}$ reviewed the implementation of decision support interventions and concluded that the factors impeding successful implementation of SDM are not yet sufficiently understood. So far, research focused mostly on physician-reported barriers and facilitators, and identified factors at the level of individuals or the patient-physician dyad (ie, the micro level). ${ }^{15}$ Recent work in the area of SDM as well as work from implementation research has emphasised the importance of also taking into account the organisational level (ie, the meso level) ${ }^{20-22}$ Qualitative studies on decision-making processes from an observers' perspective have the potential to widen the scope of research on barriers and facilitators from the micro to the meso level, but are currently lacking. As a first step, we observed multidisciplinary team meetings, which are an important component of decision-making in modern day cancer care. ${ }^{23}$ Within this study, we additionally observed decision-making processes at inpatient and outpatient clinics.

Implementation research showed that tailored implementation programmes facilitate successful implementation. Before one can develop a tailored implementation programme, a theoretical and empirical foundation should be established. ${ }^{22}$ One approach for developing a theoretically based implementation strategy is the Consolidated Framework for Implementation Research (CFIR),$^{22}$ a comprehensive framework for routine implementation in the context of health services research. It emphasises the need for a preimplementation phase to assess the current status quo before developing a tailored implementation strategy based on these data. ${ }^{22}$ Existing process evaluations revealed that for successful implementation of SDM into routine care barriers and facilitators need to be analysed. ${ }^{24}$

Thus, by observing physician-patient consultations as well as workflows (eg, ward rounds, physician-physician interactions, shift handovers) in inpatient and outpatient clinics, this study aimed to gain insight on how cancer treatment decisions are made (where, when, by whom) and to extend the understanding of decision-making beyond the dyadic physician-patient interaction.
Furthermore, this study sought to identify barriers and facilitators to the SDM process.

\section{METHODS}

\section{Study design}

A qualitative study was conducted analysing data from participant observation with a passive level of observer participation at inpatient and outpatient physicianpatient consultations as well as processes outside the patient-physician dyad (eg, physician-physician interactions, shift handovers) related to medical decision-making. ${ }^{25}$ Qualitative research using observation methodology has been shown to be useful to generate a comprehensive description of processes in clinical care. ${ }^{26}$

\section{Setting and subjects}

Data collection was carried outatinpatientwards and outpatient clinics of the University Cancer Center Hamburg (UCCH), Germany. The UCCH is a comprehensive care and research centre including all medical departments of the University Medical Center Hamburg-Eppendorf (UKE) that are involved in diagnosis and treatment of cancer. The inpatient and outpatient clinics that were included in the data were identified in cooperation with physicians at the UCCH (convenience sample). Our aim was to observe a range of diverse settings. All patients that were treated at the respective clinic within the time of the observations were asked to participate in the study. Exclusion criteria were severe cognitive impairment or insufficient German-language skills.

\section{Data collection}

Prior to observations, we contacted physicians at the inpatient wards and outpatient clinics to discuss the realisation of the observation. We also informed the nursing staff about the project.

Observations were carried out between November 2013 and January 2014 by three independent observers (PH, IS, JH (cp. acknowledgements)). PH and IS had experience in observation ${ }^{23} ; \mathrm{JH}$ had no prior experience and was briefed by IS before starting observations. Based on the CFIR framework, ${ }^{22}$ a guideline for the observations was developed by the research team prior to observations. All observers are female clinical psychologists. IS holds a $\mathrm{PhD}$ and is employed as a senior researcher, $\mathrm{PH}$ and $\mathrm{JH}$ were employed as research associates. Informed consent was obtained from all patients prior to observations. Observations at the two inpatient wards lasted for 1 week at each ward. During this week, we were present at the wards during the physicians' daytime working hours and accompanied different staff members over the course of the week, to gain insight into their workflows (eg, ward rounds, physician-physician interactions, shift handovers). At the outpatient clinics, we were present during consultation hours and accompanied seven physicians during several consultations each. 
In this academic cancer centre, setting observation of practice (eg, by undergraduate students or residents) is very common. Furthermore, physicians were only vaguely informed about the purpose of the study to minimise the probability of physicians systematically changing their behaviour due to the awareness of being observed (ie, the Hawthorne effect). We recorded our observations on a form (see online supplementary appendix 1) with prestructured sections capturing the name of the observer, time and place of the observation, a short description of the situational context and participating individuals. The form also included a section for the observation memo. This section was left unstructured in order not to limit the domains of observation. The form was designed by the principal investigator (IS), based on literature on writing field notes. ${ }^{27}$ During the consultations, we took brief notes without disturbing the usual process. We then expanded our notes after the consultations were finished. For outpatient consultations, we used one prestructured form for each consultation; for observations at inpatient wards, field notes were taken on one prestructured form for an entire day. We used field notes as we believe this method was the most suited to answer our research questions by generating data that goes beyond the consultation between the patient and physician. Also, it was less disruptive to apply in routine clinical settings than other methods like audio or video recording. During data collection, we met weekly to safeguard the quality of the observational process and its documentation in field notes. We reflected on the observation process and discussed ways to overcome challenges emerging during observations (eg, interaction with physicians, coordination of observations and note taking).

\section{Data analysis}

The handwritten field notes were digitalised and imported into MAXQDA software (VERBI, Berlin, Germany). For the digitalisation, a guideline including abbreviations the observers had used was developed. The analysis drew on principles of qualitative content analysis described by Hsieh and Shannon ${ }^{28}$ and was undertaken by two researchers. It consisted of the following steps: first, two researchers (PH and IS) read the entire set of field notes to gain an overview over the data. Second, during the initial coding process, one researcher (PH) coded about $50 \%$ of the material using a paragraph-by-paragraph approach. Third, after this phase of initial coding, comments on the material of a second researcher (IS) were compared with the established codes and the coding system was adapted. Fourth, the established codes were revised and systematised into a coding system with clusters and subcategories. Fifth, the preliminary coding system was discussed by two researchers (PH and IS) and adapted where necessary. Sixth, the remaining $50 \%$ of the material were coded by one researcher $(\mathrm{PH})$ using the established coding system. Where necessary, additional codes were created and integrated into prior codings. As a last step, the coding system was once again discussed and revised ( $\mathrm{PH}$ and
IS). During the entire coding process, we used memos to clarify codes and keep track of ideas and impressions during the process. For the presentation of the results in this paper, the themes of the qualitative analysis were organised under several headings. Those partially depict the highest order of the inductive categorization system; other headings were derived deductively from the research questions and/or theoretical background.

In addition to qualitative analysis, descriptive statistics were calculated to determine the mean duration of the consultations and the mean number of people present during consultations.

\section{RESULTS}

\section{Characteristics and description of observed consultations}

Inpatient and outpatient setting combined, a total of 119 patients were approached for this study. In total, 108 gave informed consent, 11 did not. Reasons for non-participation were, for example, not wanting to sign the informed consent form, wanting to speak to the physician alone or already participating in other studies.

Overall, $\mathrm{n}=54$ consultations with cancer patients at different outpatient clinic consultation hours at the Department of Gynaecology and the Department of Oncology of the UKE were observed. Furthermore, two inpatient wards at the Department of Oncology of the UKE were observed for 1 week each. Outpatient consultations lasted between 5 and $45 \mathrm{~min}$ (mean=17.72, $\mathrm{SD}=10.33$ ). In 26 of the 54 consultations, decisions about the primary cancer treatment (eg, chemotherapy, surgery, radiation) were made. In 13 consultations, secondary decisions related to the treatment process (eg, management of treatment-induced side effects such as pain or nausea) were made. In 15 consultations, no clinical treatment decisions were observed (eg, follow-up examination after surgery, regular follow-up care, renewal of prescription). The reasons for those consultations were, for example, appointments within the regular cycle of aftercare or to pick up new prescriptions.

Table 1 depicts the stakeholders being present at the observed outpatient consultations. In all but one consultation, a patient was present; one consultation involved a patient's family member only. In half of the consultations,

\begin{tabular}{|c|c|c|}
\hline & Frequency & $\%$ \\
\hline Patient & 53 & 98.1 \\
\hline \multicolumn{3}{|l|}{ Physicians } \\
\hline One physician & 46 & 85.2 \\
\hline Two physicians & 8 & 14.8 \\
\hline Family member(s) & 22 & 40.7 \\
\hline Nurse & 4 & 7.4 \\
\hline Medical student & 3 & 5.6 \\
\hline
\end{tabular}


solely one (or sometimes two) physician and the patient were present $(n=27,50.0 \%)$. In $40.7 \%(n=22)$ of the consultations, one or more family members were present. If nurses and/or a medical student were present, they did not take an active role in the decision-making process (ie, either no verbal interaction at all or merely involvement in the physical examination).

Regarding the observations at inpatient wards, we observed a total of 62.75 hours of physician daytime working hours. As described in the 'Methods' section, field notes were taken for an entire day each and no differentiated descriptive data that could be used for descriptive analysis were collected.

\section{Prerequisites for SDM}

In many consultations, we observed that physicians displayed patient-centred behaviour, that is, were respectful and responsive to each individual patient's needs and preferences, and taking a biopsychosocial perspective. This is a prerequisite for SDM. For example, they provided emotional support and asked their patients about treatment satisfaction. One treating physician in the outpatient setting acknowledged the patient's and the daughter's fear by saying:

I know that you always call anyways [to double check if the blood results were okay], and that is completely alright.

Furthermore, physicians' good communication skills (as described in the Kalamazoo consensus statement ${ }^{29}$ or the Calgary-Cambridge guide to the medical interview's description of the communication process ${ }^{3031}$ ), which are also an important prerequisite for SDM, were observed in some consultations. This included starting the consultation with small talk, encouraging the patient to ask questions, holding eye contact with the patients or explaining clearly. However, several other physicians did not show good communication skills by using a lot of jargon and strong wording such as 'you must', or talked about the patient rather than with the patient (outpatient as well as inpatient setting). For example, one field note says,

Senior physician used technical term several times. After about 4 to 5 times, the patient [asked]: 'Can I ask what this [term] means?

\section{Information exchange before making the decision}

Regarding the provision of information before a decision was made, physicians explained one treatment option in detail to their patients (as opposed to not explaining options in detail) in some consultations. More than one treatment option was rarely discussed in detail (ie, physicians did not give detailed information including information on risks and benefits for two or more treatment options). On several occasions, observers concluded from what they had observed that patients had not quite understood the physicians' explanations or would need more time to consider the information before making a decision. For example, regarding a newly diagnosed cancer patient, one observer noted,

Senior physician explains little, [out]patient and husband do not seem to be able to follow [the explanations].

During this consultation, the observer noted the following about the decision whether the patient should be treated with intraoperative radiation therapy,

Patient does not seem to know this option, asks about it once; physician seems to think it makes sense (does not explicitly say so $[\ldots]$ )—no detailed explanation given [of the option by the physician]

Sometimes physicians used written material or drew sketches to help patients understand the information.

In several cases, the patient and the physician talked about the patient's treatment preferences (ie, which treatment option the patient favours). This was sometimes in reaction to the patient's voicing his or her preferences, other times the physician asked for the patient's preferences. However, in none of the outpatient consultations and in only two of the inpatient consultations, the patient's participation preference (ie, whether a patient wants to be actively involved in making the decision or not) were explicitly discussed. In the two cases in which the patient's participation preference was explicitly voiced, the patients gave the power to make the decision to the physician. For example, when the physician asked, 'Do you have any more questions?', the inpatient responded, 'What questions should I ask? You do what you do. I only understand half of it anyways'.

\section{Making the decision after information exchange}

In most cases, the physicians made the treatment decisions. Either one physician made the treatment decision by himself or several physicians made medical decisions jointly (especially in inpatient wards). For example, one observer noted, 'Assistant physicians sit together [in the inpatient ward] and [informally] discuss the treatments for all patients'. Additionally, one of the observers commented, 'Most decisions during ward rounds [at inpatient wards] are taken in front of the computer before entering the patient's room'. In a few cases, decisions were made jointly, and in another few cases, patients were making the decision alone. Furthermore, in a few decision-making moments, no decision was made. In these cases, physicians either explicitly deferred the decision-making or the decision was implicitly left open.

The discussion of the patient's needs and wishes did not necessarily lead to the physician and the patient subsequently making the decision jointly. On many occasions, patients' concerns and preferences were neither explored nor included in the subsequent decision-making process, even if they were voiced by the patient during the consultation (eg, concerning artificial nutrition one [in]patient said during ward rounds 'I' $m$ not sure' and the physicians replied, 'We must do it.'). 
After the physicians had made a decision, patients agreed to the physicians' decision in many cases. For example, when it was decided that a patient should not receive a certain chemotherapeutic drug, the physician asked, 'Can you go along with this now?' and the [out]patient answered, 'Yes, this is like a Christmas present.'

\section{Involvement of third parties during the decision-making process}

Family members were observed to support the patients in the making of the decision. For example, one patient asked her daughter accompanying her during an inpatient consultation, 'Isn 't that good [treatment]?' in order to reassure herself. Additionally, one observer noted, 'Family member asked the [out]patient, if she asked all questions she had'. Also, family members were observed to support the patients outside of the patient-physician consultation. For example, one observer noted, '[in]patient wants to talk to his wife again [before making the decision]'. Besides that, family members sometimes supported in very practical ways (eg, by translating if the patient did not speak the same language as the physician or by bringing a written note with all the medication of the patient to the consultation).

The integration of nurses in the decision-making process was observed to be very limited. Nurses sometimes gave information about a patient to the physicians. They either voiced their own assessment of the patient or told the physicians what patients had said to them. One observer noted during a handover between nurses and physicians at the inpatient ward that nurses said "the patient said to me ..." or the "patient told me ..." several times'. Also, an observer wrote down that nurses "know some [in]patients from previous stays [at the hospital]' and that the nurse said, 'he always reacts to [name of drug] with [side effects]'. However, nurses were almost not at all integrated into the decision-making process.

\section{Facilitators and barriers for SDM}

The most prominent facilitator we found for SDM was if a particular patient proactively engaged himself or herself in the decision-making process. This was found to happen in three different ways: (1) the patient entered the consultation already well informed, (2) the patient asked many questions during the consultation (eg, outpatient asked 'What would happen, if I stopped taking the medication?' and physicians took the time to explain) and (3) the patient opposed a recommended treatment option leading to the necessity to revaluate and discuss other options. One observer comment said, 'Depending on the [in] patients' questions, physicians took ample time for consultation'. If a patient actively asked for involvement, physicians were found to alter their behaviour and engage with the patient in a decision-making process that was more collaborative. The few decisions that were made jointly were mostly observed when patients showed this kind of behaviour.

The main barriers to SDM found in the qualitative analyses of the field notes were (1) time pressure, (2) frequent alternation of the responsible physician (eg, due to frequent rotations of assistant physicians) and (3) poor coordination of care. Illustrating the time pressure, one outpatient said to the nurse, 'He [the physician] is always so hectic'. Also, field notes showed that the administrative work physicians had to do (eg, documentation in the electronic medical record, organising appointments for their patients at different wards) was adding even more time pressure, and that their workflows were often interrupted (eg, by their phones ringing or meetings). An example of poor coordination of care in the outpatient setting was that one observer noted, 'Who makes genetic testing? [Physician has] difficulties to find appropriate institution ([physician makes] several phone calls, internet search [during the consultation]) [...].It takes a lot of time, seems very complicated, barrier [to SDM].'

\section{DISCUSSION}

This study assessed how decisions are made in current routine cancer care using a participant observation approach. Observations of $n=54$ outpatient consultations and 62.75 hours of observations at inpatient wards were analysed using qualitative content analysis. Most of the time, either one physician alone or a group of physicians made the treatment decisions. Patients were seldom actively involved. If patients were 'active patients', this behaviour facilitated SDM. The main observed barriers were time pressure, frequent alternation of responsible physicians and poor coordination of care.

The comparison of the results of the current study with the 'three talk collaborative deliberation model' of SDM by Elwyn and colleagues ${ }^{32} 33$ shows that SDM was very rarely observed in routine cancer care. We found limited realisation of the three steps of this SDM model during our participant observations. Even if single aspects of this SDM model occurred during the observed decision-making processes, we did not observe the decision being shared between the patient and the physician when considering the whole process. Those results match existing research that also found that SDM is implemented to a low degree in routine care. ${ }^{11-14}$

We found that 'active patients' were more able to facilitate SDM. However, we cannot say why some patients were able to be more active than others. It is not a given that patients are capable of actively initiating an SDM process. Patients need to feel empowered in order to be able to actively participate in the decision-making process. ${ }^{16}$ Even affluent and highly educated patients report obstacles and concerns that keep them from openly discussing their healthcare decisions with their physician. ${ }^{17}$ This shows how difficult active involvement is for patients and emphasises the importance of patient-mediated interventions to foster SDM. One example for such an intervention is the 'Ask Three Questions' intervention, where patients are encouraged to ask their physicians three questions regarding their treatment options. ${ }^{34}$ This was shown to be associated with enhanced provision of information and the facilitation of patients' active engagement 
by physicians. ${ }^{34}$ It is worth noting that we do know from the literature that some patients do not want to be actively involved in decision-making. ${ }^{5}$ As we also know that for many patients the preferred and the perceived participation in medical decisions do not match, ${ }^{35} 36$ it would be important to explicitly assess the participation preference during consultations. However, this was almost never seen in our observations. The most prominent barriers for the implementation of SDM found in this study match barriers that have been reported in previous studies. This includes studies that assessed barriers from the physicians, ${ }^{15}$ as well as the patients' perspectives. ${ }^{16}$ It is an important task to develop and implement strategies to overcome modifiable barriers to SDM. Those strategies need to target different levels of the healthcare system (ie, individual patient or physician, teams, organisation or health policy). ${ }^{16}$ The involvement of all the members of the healthcare team into the implementation of SDM could foster more SDM in routine practice. ${ }^{11}$ For example, nurse coordinators could help overcome poor coordination of care. ${ }^{37}$

In this study, despite the potential benefit, nurses were found to play a relatively small role in current decision-making processes in routine cancer care in Germany. In other countries (such as the USA or UK), nurses have been shown to play a more active role in SDM processes in cancer care. ${ }^{38}$ The inclusion of third parties (especially nurses) into medical decision-making processes in Germany is an area that requires additional attention in the future. A possible course of action would be to transfer approaches such as nurse coordinators that are used in other countries to the German context. Also, there are interprofessional trainings that aim to support SDM as well as team communication. ${ }^{39}$

This study is an extensive qualitative exploration of decision-making processes in cancer care in a German university medical centre. To our knowledge, this is the first study that observed patient-physician consultations as well as processes related to medical decision-making beyond the dyadic relation between patient and physician. Therewith, this study gave new insights into the current state of decision-making in cancer care. It also enabled us to use these results for the development of a tailored implementation programme to foster SDM in cancer care. However, generalisability to other institutions and countries is a limitation of this study. Further research is needed to find out whether our findings are applicable to other cancer care institutions nationally and internationally. Additionally, further investigating the role of nurses and other HCPs in the decision-making process would strengthen the understanding of SDM processes in current routine care. Within our data, we have no knowledge of the staging of the patients' illness or whether a patient's case was discussed at a multidisciplinary team meeting, and therewith could not draw conclusions on the impact this might have on decision-making processes. Linking qualitative data as ours to quantitative descriptives such as clinical status, and linking data from multidisciplinary team discussions and data from patientphysician encounters would be valuable next steps. In this study, we used a solely qualitative approach. This enabled us to gather valuable in-depth information. However, the number of participants is limited. The method of participant observation enabled us to widen the focus of the observed situations as opposed to audio or video recordings of consultations. However, the field notes of our observations led to less detailed data.

\section{CONCLUSION}

This study contributed to gain further understanding of decision-making processes in routine cancer care by taking into account the physician-patient dyad as well as processes beyond the dyad. SDM was found to be very rarely implemented in current routine cancer care. Although aspects of SDM were observed on some occasions, the whole process of making medical decisions was not observed to follow the principles of SDM. While an 'active patient' was found to be a facilitator for SDM, time pressure, changes in the responsible physician and poor communication between HCPs were found to be barriers. The results of this study lay ground for the development of an implementation programme to foster SDM in routine cancer care.

Acknowledgements The authors thank Jana Hofmann for her support during data collection and Musa Cömert for the digitalisation of the field notes. They also thank Glyn Elwyn and Dominick Frosch for their advice during the course of this study.

Contributors PH was involved in planning of data collection, data collection, analysed and interpreted the data, and drafted the article. MH was involved in conception and design of the study, interpretation of the data and critical review of the manuscript. YN was involved in interpretation of the data and critical review of the manuscript. IS was involved in conception and design of the study, planning of data collection, data collection, data analysis, interpretation of the data and critical review of the manuscript. All authors reviewed the final version to be published.

Funding This work was supported by the German Research Foundation (grant number: SCHO 155/1-1).

Disclaimer The sponsors were not involved in the study design; in the collection, analysis and interpretation of data; nor in the decision to submit the paper for publication.

Competing interests $\mathrm{MH}$ declares that he is co-PI in a SDM research project funded by Mundipharma GmBH, a pharmaceutical company. IS conducted one physician training in shared-decision making within the research project funded by Mundipharma GmBH. The authors did not receive funding from Mundipharma GmBH for this paper, nor was the company involved in any steps of this study or publication process.

\section{Patient consent Obtained.}

Ethics approval The study was carried out in accordance with the Code of Ethics of the Declaration of Helsinki and was approved by the Ethics Committee of the Medical Association Hamburg (Germany).

Provenance and peer review Not commissioned; externally peer reviewed.

Data sharing statement Data (in German language only) are available upon request for researchers after consultation with the corresponding author and the responsible Ethics Committee. Please contact the corresponding author if you wish to request the data set.

Open Access This is an Open Access article distributed in accordance with the Creative Commons Attribution Non Commercial (CC BY-NC 4.0) license, which permits others to distribute, remix, adapt, build upon this work non-commercially, and license their derivative works on different terms, provided the original work is 
properly cited and the use is non-commercial. See: http://creativecommons.org/ licenses/by-nc/4.0/

(C) Article author(s) (or their employer(s) unless otherwise stated in the text of the article) 2017. All rights reserved. No commercial use is permitted unless otherwise expressly granted.

\section{REFERENCES}

1. Barry MJ, Edgman-Levitan S. Shared decision making--pinnacle of patient-centered care. N Engl J Med 2012;366:780-1.

2. Charles C, Gafni A, Whelan T. Shared decision-making in the medical encounter: what does it mean? (or it takes at least two to tango). Soc Sci Med 1997;44:681-92.

3. Härter M, van der Weijden T, Elwyn G. Policy and practice developments in the implementation of shared decision making: an international perspective. Z Evid Fortbild Qual Gesundhwes 2011;105:229-33.

4. Whitney SN. A new model of medical decisions: exploring the limits of shared decision making. Med Decis Making 2003;23:275-80.

5. Chewning B, Bylund CL, Shah B, et al. Patient preferences for shared decisions: a systematic review. Patient Educ Couns 2012;86:9-18.

6. Coulter A, Jenkinson C. European patients' views on the responsiveness of health systems and healthcare providers. Eur $J$ Public Health 2005;15:355-60.

7. Coulter A, Magee H. The European patient of the future. Maidenhead: Open University Press, 2003.

8. Leitlinienprogramm Onkologie. Interdisciplinary S3-guideline for diagnostics, treatment and aftercare of breast cancer. Berlin: AWMF, Deutschen Krebsgesellschaft e.V., Deutschen Krebshilfe e.V, 2012.

9. Bundesministerium für Gesundheit. Brochure: National Cancer Action Plan - Pheres of activity, aims, and recommendations for implementation] [German, government document. Berlin: Bundesministerium für Gesundheit, 2012.

10. Katz SJ, Hawley S. The value of sharing treatment decision making with patients: expecting too much? JAMA 2013;310:1559-60.

11. Légaré $F$, Witteman HO. Shared decision making: examining key elements and barriers to adoption into routine clinical practice. Health Aff 2013;32:276-84.

12. Charles C, Gafni A, Whelan T. Self-reported use of shared decisionmaking among breast cancer specialists and perceived barriers and facilitators to implementing this approach. Health Expect 2004;7:338-48.

13. Coulter A. Implementing shared decision making in the UK: A report for the Health Foundation. London: Health Foundation, 2009.

14. Foundation for Informed Medical Desicion Making. Informing and involving patients in medical decisions: The primary care physicians' perspective. Findings from a national survey of physicians. Boston 2009.

15. Légaré F, Ratté S, Gravel K, et al. Barriers and facilitators to implementing shared decision-making in clinical practice: update of a systematic review of health professionals' perceptions. Patient Educ Couns 2008;73:526-35.

16. Joseph-Williams N, Elwyn G, Edwards A. Knowledge is not power for patients: a systematic review and thematic synthesis of patientreported barriers and facilitators to shared decision making. Patient Educ Couns 2014;94:291-309.

17. Frosch DL, May SG, Rendle KA, et al. Authoritarian physicians and patients' fear of being labeled 'difficult' among key obstacles to shared decision making. Health Aff 2012;31:1030-8.

18. Elwyn $G$, Légaré $F$, van der Weijden $T$, et al. Arduous implementation: does the Normalisation Process Model explain why it's so difficult to embed decision support technologies for patients in routine clinical practice. Implement Sci 2008;3.57):57.

19. Elwyn G, Scholl I, Tietbohl C, et al. "Many miles to go ...": a systematic review of the implementation of patient decision support interventions into routine clinical practice. BMC Med Inform Decis Mak 2013;13:S14.

20. Müller E, Hahlweg P, Scholl I. What do stakeholders need to implement shared decision making in routine cancer care? A qualitative needs assessment. Acta Oncol 2016;55:1484-91.

21. Chaudoir SR, Dugan AG, Barr CH. Measuring factors affecting implementation of health innovations: a systematic review of structural, organizational, provider, patient, and innovation level measures. Implement Sci 2013;8:22.

22. Damschroder LJ, Aron DC, Keith RE, et al. Fostering implementation of health services research findings into practice: a consolidated framework for advancing implementation science. Implement Sci 2009;4:50.

23. Hahlweg P, Hoffmann J, Härter M, et al. In Absentia: An Exploratory Study of How Patients Are Considered in Multidisciplinary Cancer Team Meetings. PLoS One 2015;10:e0139921.

24. Elwyn G, Thomson R, et al. Implementing shared decision making in the NHS: Lessons from the Making Good Decisions in Collaboration (MAGIC) programme. In: Elwyn G, Grande S, Vidal DC, eds. Part of the routine? Case studies of implementing patient decision support into practice settings. Hanover: Dartmouth college, 2012:23-34.

25. Spradley JP. Participant observation. New York: Holt, Rinehart and Winston, 1980.

26. Savage J. Ethnography and health care. BMJ 2000;321:1400-2.

27. Emerson RM, Fretz RI, Shaw LL. Writing ethnographic fieldnotes. Second Edition. Chicago: edThe University of Chicago Press;, 2011.

28. Hsieh HF, Shannon SE. Three approaches to qualitative content analysis. Qual Health Res 2005;15:1277-88.

29. Makoul G. Essential elements of communication in medical encounters: the Kalamazoo consensus statement. Acad Med 2001;76:390-3

30. Kurtz S, Silverman J, Draper J. Teaching and Learning Communication Skills in Medicine. Oxford: Radcliffe Medical Press, 1998.

31. Silverman J, Kurtz S, Draper J. Skills for Communicating with Patients. Radcliffe Medical Press: Oxford, 1998.

32. Elwyn G, Frosch D, Thomson R, et al. Shared decision making: a model for clinical practice. J Gen Intern Med 2012;27:1361-7.

33. Elwyn G, Tsulukidze M, Edwards A, et al. Using a 'talk' model of shared decision making to propose an observation-based measure: Observer OPTION 5 Item. Patient Educ Couns 2013;93:265-71.

34. Shepherd HL, Barratt A, Trevena LJ, et al. Three questions that patients can ask to improve the quality of information physicians give about treatment options: a cross-over trial. Patient Educ Couns 2011;84:379-85.

35. Brom L, Hopmans W, Pasman HR, et al. Congruence between patients' preferred and perceived participation in medical decisionmaking: a review of the literature. BMC Med Inform Decis Mak 2014;14:25.

36. Tariman JD, Berry DL, Cochrane B, et al. Preferred and actual participation roles during health care decision making in persons with cancer: a systematic review. Ann Oncol 2010;21:1145-51.

37. Nutt M, Hungerford C. Nurse care coordinators: definitions and scope of practice. Contemp Nurse 2010;36:71-81.

38. Tariman JD, Mehmeti E, Spawn N, et al. Oncology Nursing and Shared Decision Making for Cancer Treatment. Clin J Oncol Nurs 2016;20:560-3.

39. Koerner M, Wirtz M, Michaelis M, et al. A multicentre clusterrandomized controlled study to evaluate a train-the-trainer programme for implementing internal and external participation in medical rehabilitation. Clin Rehabil 2014;28:20-35. 